\title{
Localized Spoof Plasmons Arise while Texturing Closed Surfaces
}

\author{
Anders Pors, ${ }^{1,2, *}$ Esteban Moreno, ${ }^{1}$ L. Martin-Moreno, ${ }^{3}$ J. B. Pendry, ${ }^{4}$ and F. J. Garcia-Vidal ${ }^{1, \dagger}$ \\ ${ }^{1}$ Departamento de Física Teórica de la Materia Condensada, Universidad Autónoma de Madrid, E-28049 Madrid, Spain \\ ${ }^{2}$ Mads Clausen Institute (MCI), University of Southern Denmark, Alsion 2, DK-6400 Sønderborg, Denmark \\ ${ }^{3}$ Instituto de Ciencia de Materiales de Aragón and Departamento de Física de la Materia Condensada, \\ CSIC-Universidad de Zaragoza, E-50009 Zaragoza, Spain \\ ${ }^{4}$ Imperial College London, Department of Physics, The Blackett Laboratory, London SW7 2AZ, United Kingdom
}

(Received 4 January 2012; published 31 May 2012)

\begin{abstract}
We demonstrate that textured closed surfaces, i.e., particles made of perfect electric conductors (PECs), are able to support localized electromagnetic resonances with properties resembling those of localized surface plasmons (LSPs) in the optical regime. Because of their similar behavior, we name these types of resonances as spoof LSPs. As a way of example, we show the existence of spoof LSPs in periodically textured PEC cylinders and the almost perfect analogy to optical plasmonics. We also present a metamaterial approach that captures the basic ingredients of their electromagnetic response.
\end{abstract}

In recent years, plasmonics has attracted a great deal of attention due to the possibility of subwavelength confinement and concomitant enhancement of the electromagnetic (EM) field at metal-dielectric interfaces [1-3]. Surface plasmons exist either as propagating surface plasmon polaritons (SPPs) for extended interfaces and waveguide configurations or as localized surface plasmons (LSPs) in structures with closed surfaces, i.e., particles [4]. Common to these surface modes is a list of promising applications taking advantage of their subwavelength nature. For example, SPPs are excellent candidates for miniaturization of photonic circuits [5], whereas LSPs have been used in near-field optics [6], surface-enhanced spectroscopy [7-9], plasmonic antennas [10,11], and photovoltaics $[12,13]$ due to the field enhancement (FE) occurring at the LSP resonance. On a more fundamental level, it has been shown that both LSPs and SPPs are able to enhance light-matter interaction at the subwavelength scale $[14,15]$. As subwavelength confinement of surface plasmons relies on the penetration of the EM field into the metal, its usage is inevitably limited to visible or near-infrared frequencies. At lower frequencies, metals sustain EM modes that are weakly bound and thus behave akin to perfect electric conductors (PECs), which do not support surface plasmons.

To export the exciting properties of surface plasmons to low frequencies (far infrared, terahertz or microwave), the concept of spoof surface plasmons was developed [16,17]. In contrast to conventional SPPs, spoof surface plasmons also exist in the PEC limit since they are geometry-induced bound EM modes that arise when a metal surface is periodically textured on a subwavelength scale. Spoof SPPs with subwavelength transverse confinement have been found both in periodic perforated plane surfaces [16-20] and in a variety of structured waveguide configurations [21-24]. In this Letter, we extend the concept of designer plasmons by demonstrating the existence of spoof localized surface plasmons (spoof-LSPs) in periodically textured PEC particles both in two and three dimensions. To illustrate our finding, we show that an almost perfect analogy exists between the EM response of a structured two-dimensional (2D) PEC cylinder and that of a regular metallic cylinder with a Drude dielectric permittivity. In this way, it is expected that all the capabilities found for LSP resonances in the optical regime can be directly transferred to lower frequencies thanks to the existence of spoof LSP modes.

As a way of example, we first consider a textured 2D PEC cylinder [Fig. 1(a)]. The structure consists of an inner cylinder of radius $r$ overlaid with radial grooves of height $h=R-r$, width $a$, and periodicity $d=2 \pi R / N$, where $N$ is the total number of grooves. The number of grooves and the radius of the cylinder are chosen to satisfy $d \ll \lambda_{0}\left(\lambda_{0}\right.$ is the wavelength of the incident wave). The grooves are filled with a dielectric of refractive index $n_{g}$, and the
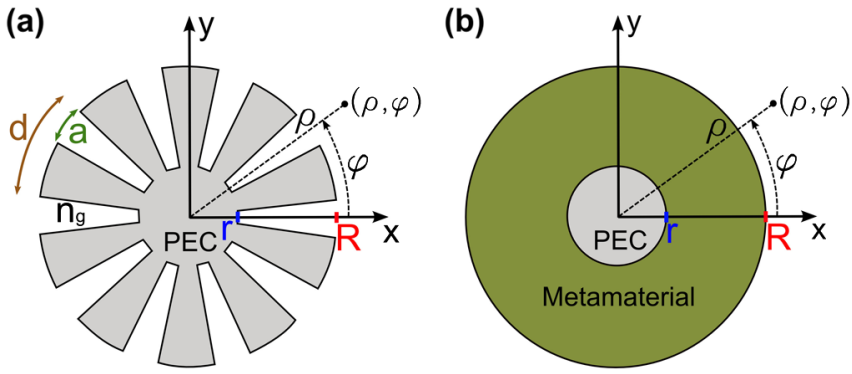

FIG. 1 (color online). (a) A two-dimensional corrugated PEC cylinder (invariant along the $z$ direction) with the inner and outer radii $r$ and $R$, periodicity $d$, and groove width $a$. The refractive index in the grooves is denoted $n_{g}$. (b) In the effective medium approximation the geometry displayed in (a) behaves as an inhomogeneous and anisotropic layer of thickness $(R-r)$ wrapped around a PEC cylinder of radius $r$. 
surrounding medium is assumed to be air. Because the resonance frequencies scale with the reciprocal size of the structure within the PEC approximation, the outer radius $R$ is used as the unit length. In order to probe the plasmonic resonances, we consider in the following a TM-polarized incident plane wave ( $\vec{H}$ pointing along the $z$ direction) propagating along the $-y$ axis. One should note in passing that related structures have been studied in the past in connection with antenna design $[25,26]$, but the analogy to optical plasmonics has been never realized.

The EM response of particles without Ohmic losses is conveniently described by the scattering cross section (SCS). In Fig. 2(b) (solid line), we present the normalized SCS as a function of the normalized frequency for a representative textured PEC cylinder $(r=0.33 R, N=60$, $a=0.4 d, n_{g}=1$, and $\omega_{a} R=0.89 \pi c$, where $c$ is the velocity of light in vacuum) calculated using the commercial software COMSOL MULTIPHYSICS. The SCS is normalized to the diameter $2 R$, whereas the frequency is

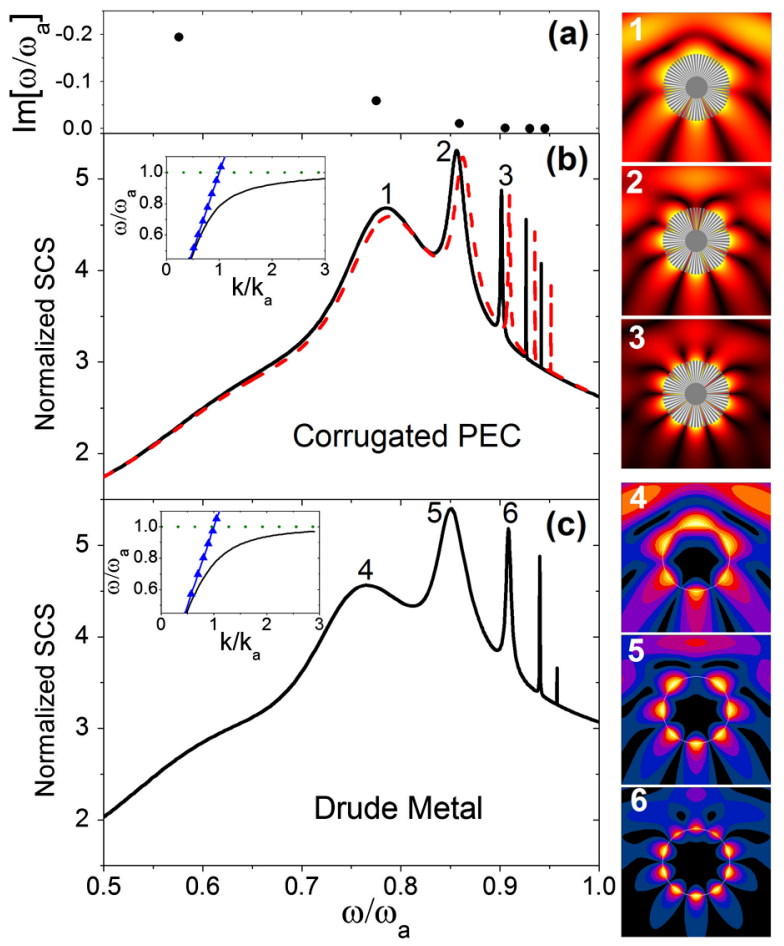

FIG. 2 (color online). (a) Complex resonance frequencies found using the modal expansion technique and (b) the numerically computed SCS (solid line) for a textured PEC cylinder with $r=0.33 R, N=60, a=0.4 d, n_{g}=1$, and $\omega_{a} R=0.89 \pi c$. The dashed line in (b) corresponds to the calculation in the metamaterial approximation (see main text). The inset shows the dispersion relation of the corresponding spoof SPP. (c) SCS for a Drude metal cylinder of radius $R$ with the same $\omega_{a} R$-value as in (b). The inset shows the dispersion relation for Drude metal SPPs. The right panels display the absolute $H$ field at the hexa-, octo-, and decapole resonances for the two systems. The color scales are chosen so that the field outside the textured cylinder is emphasized. normalized to the asymptotic frequency $\omega_{a}$ of the corresponding spoof SPP that would propagate on a periodically textured flat surface with similar grooves. The inset of Fig. 2(b) shows the dispersion relation of these spoof SPPs (solid line), which has been obtained with the multiple multipole method [27], and the free-space light dispersion (triangular dotted line) in normalized units $\left(k_{a}=\omega_{a} / c\right)$. The asymptote frequency is mainly controlled by the groove height $h$ and refractive index $n_{g}$ as $\omega_{a} \approx \pi c /\left(2 h n_{g}\right)$ [17].

The SCS spectrum shown in Fig. 2(b) consists of multiple peaks that must be related to a resonant EM behavior of the corrugated PEC cylinder. The physics behind this spectrum is uncovered by a direct comparison with the optical response of a simple metallic cylinder having the Drude permittivity $\epsilon_{m}(\omega)=1-\omega_{p}^{2} / \omega^{2}$, where $\omega_{p}$ is the plasma frequency of the metal. Note that we have neglected absorption in the Drude permittivity to be in accordance with the PEC approximation. In Fig. 2(c) we present the SCS of a metal cylinder with the same $\omega_{a} R$-value $\left(\omega_{a}=\omega_{p} / \sqrt{2}\right)$ as in Fig. 2(b). Despite the very different structures and frequency regimes, it is remarkable how the textured PEC cylinder mimics in spectral position, value, and resonant linewidth the plasmonic response of a Drude cylinder. The reason is the existence of spoof LSPs that emulate conventional LSPs. This is also clearly seen in the right panels of Fig. 2, which display the magnetic field amplitude at the hexa-, octo-, and decapole resonances when excited by a TM-polarized plane wave. The fields outside the textured PEC cylinder and Drude cylinder $(\rho>R)$ are indeed very similar. It can be demonstrated that the compelling analogy between spoof LSPs and optical LSPs can be traced back to the similarity in the dispersion relations of spoof SPPs and Drude metal SPPs, as shown in the insets of Figs. 2(b) and 2(c).

In order to gain a deeper insight into the origin of spoof LSPs, we present an analytical equation for the resonance condition of spoof LSPs in textured cylinders following the steps of the modal expansion technique. First, we write the magnetic $H_{z}$ component (TM wave) outside the textured cylinder as [harmonic time: $\exp (-i \omega t)]$

$$
H_{z}^{\text {out }}=\sum_{n=-\infty}^{\infty} A_{n} H_{n}^{(1)}\left(k_{0} \rho\right) e^{i n \varphi},
$$

where $A_{n}$ are constants, $H_{n}^{(1)}$ is the Hankel function of first kind and order $n, k_{0}$ is the wave number in air, and $(\rho, \varphi)$ are the polar coordinates [Fig. 1(a)]. Note that diffraction has been neglected when writing Eq. (1), which is a good approximation for $d \ll \lambda_{0}$. Second, since the grooves are subwavelength, only the fundamental radial waveguide mode is considered in the expansion of the EM fields within the grooves:

$$
H_{z}^{g}=B^{+} H_{0}^{(1)}\left(k_{0} n_{g} \rho\right)+B^{-} H_{0}^{(2)}\left(k_{0} n_{g} \rho\right),
$$


where $B^{+}$and $B^{-}$are constants with $H_{0}^{(1)}$ and $H_{0}^{(2)}$ being the radially outward and inward propagating modes, respectively. The resonance condition for the individual modes is obtained by applying matching boundary conditions on $H_{z}$ and $E_{\varphi}$ for a given $n$ in Eqs. (1) and (2). By doing so, we derive the following transcendental equation:

$$
S_{n}^{2} \frac{H_{n}^{(1)}\left(k_{0} R\right)}{H_{n}^{(1)^{\prime}}\left(k_{0} R\right)} \frac{f}{g}=n_{g},
$$

where $\quad f=J_{1}\left(k_{0} n_{g} r\right) Y_{1}\left(k_{0} n_{g} R\right)-J_{1}\left(k_{0} n_{g} R\right) Y_{1}\left(k_{0} n_{g} r\right)$ and $g=J_{0}\left(k_{0} n_{g} R\right) Y_{1}\left(k_{0} n_{g} r\right)-J_{1}\left(k_{0} n_{g} r\right) Y_{0}\left(k_{0} n_{g} R\right), \quad J_{0}$ and $J_{1}\left(Y_{0}\right.$ and $\left.Y_{1}\right)$ being the zero and first order Bessel functions of first (second) kind, respectively, $n$ is the mode number (e.g., $n=1$ is the dipole mode), and $S_{n}$ is given by $S_{n}=\sqrt{a / d} \operatorname{sinc}(n a /(2 R))$. The ' means differentiation with respect to the argument in parenthesis. It is worth noting that all details about the unit cell are found in $S_{n}$, which, for subwavelength texturing $(a \ll R)$, approximates to $S_{n} \simeq \sqrt{a / d}$. Figure 2(a) shows the complex resonance frequencies calculated using Eq. (3) for the same structure as in Fig. 2(b). The predictions of the analytical model are in very good agreement with the numerical simulations: resonances that are too far away from the real frequency axis are only barely apparent in the SCS, whereas complex resonance frequencies with a small imaginary part show up in the SCS as extremely narrow peaks. This model and the corresponding resonance condition [Eq. (3)] are consistent with a picture in which an EM mode is running around the cylinder surface with resonances emerging when an integer number of modal wavelengths fit into the perimeter.

The analogy between the EM response of a corrugated PEC cylinder and that of a metallic cylinder at optical frequencies is very insightful, but it is not able to describe accurately the behavior of the EM fields inside the grooves. The reason is that in a real metal the EM fields decay exponentially inside the metal, whereas in a corrugated PEC cylinder the existence of a propagating mode inside the grooves leads to EM fields that are not evanescent. In order to complete the picture, here we develop a complementary way to look into the physics of the spoof LSPs by applying the concept of an EM metamaterial. As we are texturing the PEC cylinder on a subwavelength scale $(d \ll$ $\lambda_{0}$ ), the incident field does not feel the details of the structure. Consequently, it is expected that the region with grooves can be interpreted as an effective medium of thickness $h$ as illustrated in Fig. 1(b). The effective parameters can be deduced from the following line of thought: first, as the PEC boundary condition in the subwavelength radial grooves forces $E_{\rho}=E_{z}=0$ and $H_{\varphi}=0, \epsilon_{\rho}=\epsilon_{z}=-\infty$ and $\mu_{\varphi}=\infty$. By averaging $1 / \epsilon$ in the $\varphi$ direction, we obtain $\epsilon_{\varphi}=n_{g}^{2} d / a$. As light propagates in the grooves in the $\rho$ or $z$ directions with the velocity $c / n_{g}$, the effective material must satisfy the equa- tions $\sqrt{\epsilon_{\varphi} \mu_{z}}=\sqrt{\epsilon_{\varphi} \mu_{\rho}}=n_{g}$, leading to $\mu_{\rho}=\mu_{z}=a / d$. Note that in Cartesian coordinates these effective parameters transform to permittivity and permeability tensors with off-diagonal elements and elements that depend on the $(x, y)$ coordinates. Put differently, in the metamaterial approximation the subwavelength grooves behave as an anisotropic and inhomogeneous layer with both electric and magnetic properties. It is important to note that this metamaterial, which combines positive and negative values for the permittivity and permeability tensors, supports the propagation of spoof SPPs that present a plasmonlike dispersion relation as those shown in Figs. 2(b) and 2(c).

The metamaterial approximation also has the advantage of an analytical treatment due to the simpler geometry [Fig. 1(b)]. In fact, it is possible by straightforward algebra to derive the following analytic expression for the SCS, $\sigma_{\mathrm{sc}}$ :

$$
\sigma_{\mathrm{sc}}=\frac{4 c}{\omega} \sum_{n=-\infty}^{\infty}\left|C_{n}\right|^{2}
$$

where

$$
C_{n}=-i^{n} \frac{\frac{a}{d} J_{n}\left(k_{0} R\right) f-n_{g} J_{n}^{\prime}\left(k_{0} R\right) g}{\frac{a}{d} H_{n}^{(1)}\left(k_{0} R\right) f-n_{g} H_{n}^{(1)^{\prime}}\left(k_{0} R\right) g} .
$$

It is evident from Eq. (5) that the resonance condition (zero in the denominator) is identical to Eq. (3), just with $S_{n}=\sqrt{a / d}$. Hence, the metamaterial approximation is virtually exact for the case of very narrow grooves $(a \ll R)$. The validity of the metamaterial approximation has been verified by calculating the SCS of the structure in Fig. 1(b) using Eq. (4). The result is shown in Fig. 2(b) (dashed line) and it is clear that the metamaterial approximation captures all the resonant features of the spectrum. An important asset of the metamaterial approach is that it simplifies the calculation of the EM fields of corrugated PEC particles in complex arrangements.

The probably best known case in particle plasmonics is the excitation of LSPs in subwavelength particles at which only the dipole mode is excited (i.e., the quasistatic limit). A similar situation can be found in textured PEC particles, but, in contrast to optical plasmonics, the subwavelength regime cannot be reached by just scaling down the structure since resonance frequencies scale accordingly within the PEC approximation. Therefore, the subwavelength regime can only be achieved by lowering $\omega_{a}$ (while keeping $R$ constant) which corresponds to increasing $h$ and/or $n_{g}$, as discussed previously. For simplicity, in this proof-ofprinciple study, we increase the refractive index within the grooves, although structures with elongated (i.e., curved) grooves are also feasible alternatives. Figure 3 shows the SCS of a subwavelength textured PEC cylinder $(r=0.4 R$, $N=30, a=0.8 d$ ) for which $n_{g}=8$ and $\omega_{a} R=0.12 \pi c$. By looking at the resonant $H$ field (insets of Fig. 3) it is evident that the dominant peak corresponds to the dipole 


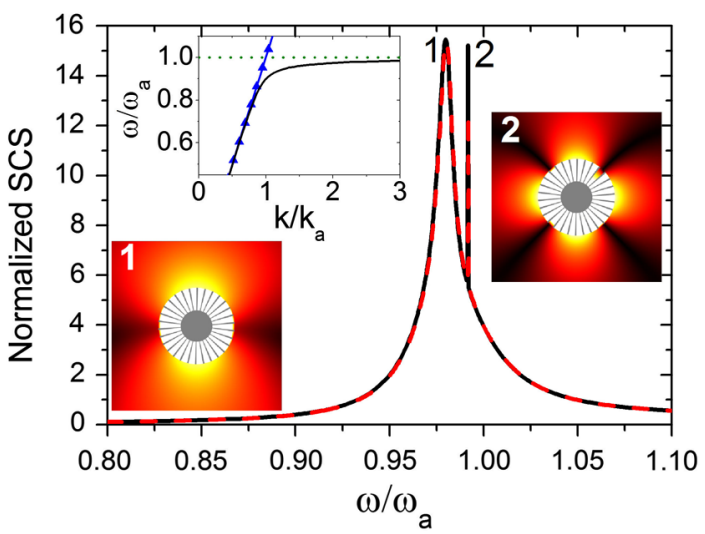

FIG. 3 (color online). SCS for a subwavelength corrugated PEC cylinder with $r=0.4 R, N=30, a=0.8 d, n_{g}=8$, and $\omega_{a} R=0.12 \pi c$. The dashed line corresponds to the metamaterial approximation. The insets show the corresponding dispersion relation for the spoof SPP and magnetic field amplitude plots at the di- and quadrupole resonances. The color scales are chosen so that the field outside the textured cylinder is emphasized.

mode while the sharp peak is identified as the quadrupole mode. One should note that the quadrupole mode will, as opposed to the dipole mode, disappear if Ohmic losses are introduced into the metal due to its extremely narrow linewidth. The spoof SPP dispersion relation for the considered structure (upper left inset Fig. 3) shows a more abrupt behavior compared to the SPP dispersion in Drude metals [inset of Fig. 2(c)], thus explaining why in the subwavelength case the resonant peaks are very narrow. It is also apparent from Fig. 3 that the metamaterial approximation (dashed line) works better for this very deep subwavelength structure than for the case of a large cylinder displayed in Fig. 2.

Up to now, we have analyzed the EM response of corrugated particles within the PEC approximation. As discussed in the Supplemental Material [28], when metal losses are introduced into the picture, the main spoof LSP resonances appearing in the scattering spectra are broadened, whereas the very sharp high-order resonances tend to be suppressed. This is exactly the behavior observed for conventional LSPs in the optical regime when Ohmic losses are incorporated into the metal permittivity.

Finally, we address the issue whether spoof LSPs also arise in three-dimensional structures. Here we demonstrate how the spoof dipole resonance of two interacting threedimensional textured cylinders can be used to enhance the electric field in the center of the gap. A sketch of the system is shown in Fig. 4(a), where the cylinders are now also characterized by the height $L$ and the mutual separation $s$. The incident wave is assumed to be $x$ polarized and propagates along the $z$ direction. Compared to the 2D structure, the excitation configuration is different, but this does not prevent us from exciting the electric dipole mode. (a)
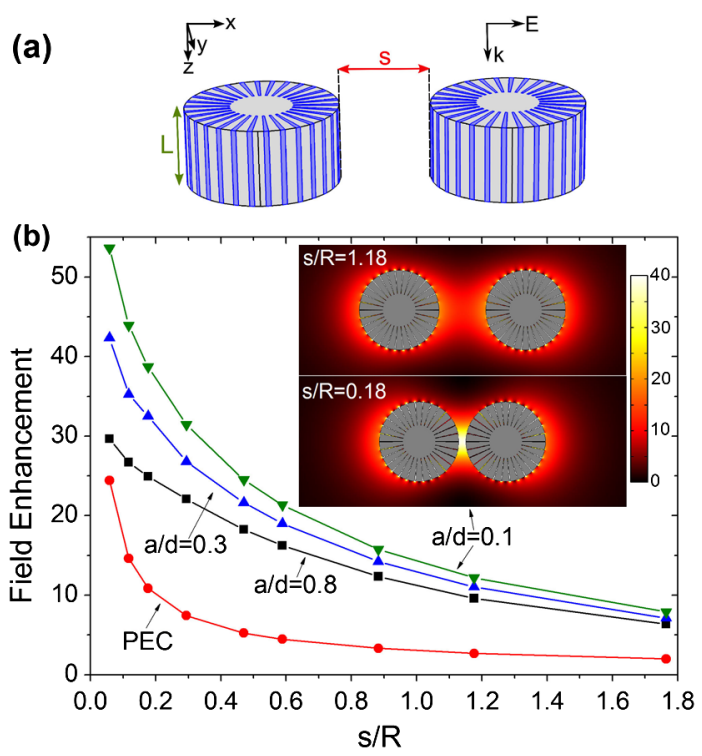

FIG. 4 (color online). (a) Two three-dimensional corrugated PEC cylinders with height $L$ and separation $s$. The incident plane wave is $x$ polarized and propagating along the $z$ direction. (b) Electric field enhancement in the center of the gap as a function of the separation for corrugated PEC cylinders with $r=0.4 R, N=30, n_{g}=8, L=R$ and different values of $a / d$. The field enhancement is evaluated at the dipole resonance frequency of the combined system. The line with circular dots corresponds to PEC cylinders without corrugation. The inset shows the field enhancement in the $x y$ plane for $a / d=0.1$ at two different separations.

Figure 4(b) shows the FE as a function of separation for three different values of $a / d$, with $r=0.4 R, N=30$, $n_{g}=8$, and $L=R$. Although not shown, the metamaterial approach described before is able to reproduce accurately the evolution of the FE with both $s / R$ and $a / d$. FE is evaluated at the dipole resonance of the combined system. Importantly, we see a significant increase in FE for decreasing separation (insets of Fig. 4). The most important property of the system is the plasmonic effect (spoof LSP), which largely increases the FE compared to the FE of a system of two noncorrugated PEC cylinders (line with circular dots). Notice that the interaction between two textured cylinders is presented here as a proof of concept, and therefore it is very likely that other textured geometries would produce larger FEs. We have also analyzed the effect of Ohmic losses on the FE associated with spoof LSPs (details can be found in the Supplemental Material [28]). As expected, these absorption losses reduce the level of FE at the dipole resonance observed in Fig. 4(b). However, the corrugated cylinder always displays higher FE than that of noncorrugated metal cylinders, hereby proving a potential use of spoof LSPs for low frequencies. Moreover, our investigation also shows that the system under study can be advantageously exploited up to the infrared regime $(\sim 100 \mathrm{THz})$, where the increased FE is 
related to the hybridization between spoof LSPs and conventional LSPs.

In conclusion, we have shown that although metallic particles do not display surface plasmon resonances at low frequencies, they may be forced to do so by incorporating a periodic corrugation to their surfaces. An effective medium analytical approach can be used to completely understand the behavior of the textured particle. The spoof localized surface plasmon modes created by the periodic modulation mimic all aspects of conventional localized plasmon resonances. Thanks to this new concept, a whole bunch of phenomena already known to work for visible and near-infrared frequencies can be safely transferred to lower frequencies. This finding may have a very significant impact on both fundamental and applied research.

*pors@mci.sdu.dk †j.garcia@uam.es

[1] W. L. Barnes, A. Dereux, and T. W. Ebbesen, Nature (London) 424, 824 (2003).

[2] E. Ozbay, Science 311, 189 (2006).

[3] S. A. Maier, Plasmonics: Fundamentals and Applications (Springer, New York, 2007).

[4] U. Kreibig and M. Vollmer, Optical Properties of Metal Clusters (Springer, Berlin, 1995).

[5] T.W. Ebbesen, C. Genet, and S. I. Bozhevolnyi, Phys. Today 61, No. 5, 44 (2008).

[6] L. Novotny and B. Hecht, Principles of Nano-Optics (Cambridge University Press, Cambridge, 2006).

[7] F. J. García-Vidal and J. B. Pendry, Phys. Rev. Lett. 77, 1163 (1996).

[8] E. Prodan, C. Radloff, N. J. Halas, and P. Nordlander, Science 302, 419 (2003).

[9] J. N. Anker, W. P. Hall, O. Lyandres, N. Shah, J. Zhao, and R. P. V. Duyne, Nature Mater. 7, 442 (2008).

[10] E. Cubukcu, E. A. Kort, K. B. Crozier, and F. Capasso, Appl. Phys. Lett. 89, 093120 (2006).
[11] M. Schnell, A. García-Etxarri, A. J. Hubert, K. Crozier, J. Aizpurua, and R. Hillenbrand, Nature Photon. 3, 287 (2009).

[12] H. A. Atwater and A. Polman, Nature Mater. 9, 205 (2010).

[13] J. A. Schuller, E. S. Barnard, W. Cai, Y. C. Jun, J. S. White, and M. L. Brongersma, Nature Mater. 9, 193 (2010).

[14] A. V. Akimov, A. Mukherjee, C. L. Yu, D. E. Chang, A. S. Zibrov, P. R. Hemmer, H. Park, and M. D. Lukin, Nature (London) 450, 402 (2007).

[15] A. Trügler and U. Hohenester, Phys. Rev. B 77, 115403 (2008).

[16] J. B. Pendry, L. Martín-Moreno, and F. J. García-Vidal, Science 305, 847 (2004).

[17] F. J. García-Vidal, L. Martín-Moreno, and J. B. Pendry, J. Opt. A 7, S97 (2005).

[18] A. P. Hibbins, B. R. Evans, and J. R. Sambles, Science 308, 670 (2005).

[19] F. J. García de Abajo and J. J. Sáenz, Phys. Rev. Lett. 95, 233901 (2005).

[20] N. Yu, Q. J. Wang, M. A. Kats, J. A. Fan, S. P. Khanna, L. Li, A. G. Davies, E. H. Linfield, and F. Capasso, Nature Mater. 9, 730 (2010).

[21] S. A. Maier, S. R. Andrews, L. Martín-Moreno, and F. J. García-Vidal, Phys. Rev. Lett. 97, 176805 (2006).

[22] D. Martin-Cano, M.L. Nesterov, A. I. FernandezDominguez, F. J. García-Vidal, L. Martín-Moreno, and E. Moreno, Opt. Express 18, 754 (2010).

[23] M. A. Kats, D. Woolf, R. Blanchard, N. Yu, and F. Capasso, Opt. Express 19, 14860 (2011).

[24] E. M. G. Brock, E. Hendry, and A. P. Hibbins, Appl. Phys. Lett. 99, 051108 (2011).

[25] J.R. Wait, IEEE Trans. Antennas Propag. 9, 330 (1961).

[26] G. Manara, A. Monorchio, and G. Pelosi, IEEE Trans. Antennas Propag. 45, 1700 (1997).

[27] C. Hafner, Post-Modern Electromagnetics (Wiley, Chichester, 1999).

[28] See Supplemental Material at http://link.aps.org/ supplemental/10.1103/PhysRevLett.108.223905 for a discussion of additional results when metal dissipation is taken into account. 